\title{
Repeated Social Stress Increases Reward Salience and Impairs Encoding of Prediction by Rat Locus Coeruleus Neurons
}

\author{
Nayla N Chaijale', Kevin Snyder², Jay Arner', Andre L Curtis' and Rita J Valentino*, \\ 'Department of Anesthesiology and Critical Care Medicine, The Children's Hospital of Philadelphia, Philadelphia, PA, USA; ${ }^{2}$ Neuroscience \\ Graduate Group, The University of Pennsylvania, Philadelphia, PA, USA
}

\begin{abstract}
Stress is implicated in psychopathology characterized by cognitive dysfunction. Cognitive responses to stress are regulated by the locus coeruleus-norepinephrine (LC-NE) system. As social stress is a prevalent human stressor, this study determined the impact of repeated social stress on the relationship between LC neuronal activity and behavior during the performance of cognitive tasks. Social stressexposed rats performed better at intradimensional set shifting (IDS) and made fewer perseverative errors during reversal learning (REV). LC neurons of control rats were task responsive, being activated after the choice and before reward. Social stress shifted LC neuronal activity from being task responsive to being reward responsive during IDS and REV. LC neurons of stressed rats were activated by reward and tonically inhibited by reward omission with incorrect choices. In contrast, LC neurons of stress-naive rats were only tonically inhibited by reward omission. Reward-related LC activation in stressed rats was unrelated to predictability because it did not habituate as learning progressed. The findings suggest that social stress history increases reward salience and impairs processes that compute predictability for LC neurons. These effects of social stress on LC neuronal activity could facilitate learning as indicated by improved performance in stressed rats. However, the ability of social stress history to enhance responses to behavioral outcomes may have a role in the association between stress and addictive behaviors. In addition, magnified fluctuations in LC activity in response to opposing behavioral consequences may underlie volatile changes in emotional arousal that characterize post-traumatic stress disorder.

Neuropsychopharmacology (20I5) 40, 5I3-523; doi:I0.1038/npp.20I4.200; published online 3 September 20I4
\end{abstract}

In response to acute stressors, neural systems that regulate the hypothalamic-pituitary-adrenal axis, autonomic function, behavior, and cognition are engaged in a coordinated manner to cope with the stressor and promote survival. Persistent or repeated activation of these stress systems as a result of chronic or repeated stress, or inappropriate activation of the systems in the absence of stress, is maladaptive and results in pathology (Chrousos and Gold, 1992; de Kloet et al, 2005; McEwen, 1998). Such maladaptive stress responses are thought to underlie symptoms such as hyperarousal, inappropriate fear, and attentional and cognitive dysfunctions that characterize certain psychiatric disorders (Gold and Chrousos, 2002). Interestingly, although many studies report impairment of cognitive processes by stressors, there are also reports of enhancement and these differences may depend on the specific stressor and the cognitive endpoint (Bondi et al, 2008; Butts et al, 2013; Graybeal et al, 2011; Lapiz-Bluhm et al, 2009; Thai et al, 2013). These differences underscore the complexity of effects of stress on neural circuits that regulate cognitive functions.

*Correspondence: Dr RJ Valentino, Department of Anesthesiology and Critical Care Medicine, The Children's Hospital of Philadelphia, 3416 Civic Center Boulevard, Abramson Pediatric Research Center Rm 402, Philadelphia, PA 19104, USA, Tel: +215 590 0650, Fax: +2I5 590 3364, E-mail: rjv@mail.med.upenn.edu

Received I3 May 20 I4; revised 16 July 20 I4; accepted 2 August 20 I4; accepted article preview online I| August 2014
The locus coeruleus-norepinephrine (LC-NE) system is a stress-response system that has been implicated in cognitive responses to stress and in stress-related psychopathology (Southwick et al, 1999; Valentino and Van Bockstaele, 2008; Wong et al, 2000). LC neurons are spontaneously active and their discharge rate is positively correlated to behavioral indices of arousal (Aston-Jones and Bloom, 1981b; Foote et al, 1980). Salient stimuli elicit a phasic activation of LC neurons that precedes orientation to the stimulus and this has implicated the system in attention (Aston-Jones and Bloom, 1981a; Berridge and Waterhouse, 2003; Foote et al, 1980). LC neuronal recordings in animals performing operant tasks indicate that phasic LC discharge is associated with focused attention and staying 'on-task', whereas hightonic LC discharge is associated with labile attention and task disengagement (Aston-Jones and Cohen, 2005; Bouret and Sara, 2005; Sara, 2009). It has been suggested that this high-tonic mode of LC discharge facilitates cognitive flexibility (Aston-Jones and Cohen, 2005). Acute stressors and exposure to the stress-related peptide, corticotropinreleasing factor (CRF) bias LC activity towards a high-tonic state that may be adaptive in a dynamic environment with life-threatening challenges (Curtis et al, 2012; Valentino and Foote, 1987; Valentino and Wehby, 1988). Notably, certain doses of CRF improve extradimensional set-shifting performance during an attentional set-shifting task (AST), an endpoint of cognitive flexibility (Snyder et al, 2012). In contrast to the activating effects of acute stressors on 
LC neuronal activity, repeated social stress produces an enduring inhibition of rat LC discharge that is apparent several days after the last stressor (Chaijale et al, 2013). These findings are relevant to humans given that social stress is one of the most common human stressors. A similar enduring inhibition of LC activity occurs in rats exposed to a model of post-traumatic stress disorder that involves the administration of three consecutive stressors (George et al, 2013). It is unknown how this state of LC inhibition impacts on cognition.

As cognitive dysfunction is thought to be one of the maladaptive consequences of repeated stress and the LC-NE system has been implicated in cognitive aspects of the stress response, the present study investigated the long-term effects of repeated social stress on LC activity recorded during the performance of an AST that assesses simple discrimination (SD), compound discrimination (CD), intradimensional set shifting (IDS), reversal learning (REV), and extradimensional set shifting. The results provided evidence that repeated social stress changes the relationship between LC neuronal activity and task performance, and renders LC neurons responsive to reward regardless of its predictability.

\section{MATERIALS AND METHODS}

\section{Animals}

Adult male Sprague-Dawley rats were used as intruders or matched controls (275-300 g) and male Long-Evans (650$850 \mathrm{~g}$ ) retired breeders were used as residents (Charles River, Wilmington, MA). Rats were individually housed in a controlled environment $\left(20^{\circ} \mathrm{C}, 12 \mathrm{~h}\right.$ light/dark cycle, lights on at $0700 \mathrm{~h}$ ). Food and water were available ad libitum unless noted. Care and use of animals was approved by the Children's Hospital of Philadelphia Institutional Animal Care and Use Committee and in accordance with NIH Guide for the Care and Use of Laboratory Animals.

\section{Surgery and Stress}

Figure 1al shows an overall timeline of the experimental protocol. All rats used as intruders or matched controls were anesthetized, positioned in a stereotaxic instrument, and surgically prepared for localization of the LC and subsequent implantation of an eight-wire microelectrode array for neuronal recordings as previously described (Curtis et al, 2012). Following recovery, rats were randomly assigned to receive control manipulation or social stress in the resident-intruder model of social stress (Chaijale et al, 2013; Miczek, 1979). Intruder rats were placed into the cage of a resident rat. A period of investigation was typically followed by an aggressive encounter initiated by the resident. When the intruder either assumed a supine posture signaling subordination (defeat) or $15 \mathrm{~min}$ had elapsed from the time when the intruder was placed into the cage, the intruder and resident were separated by a wire mesh partition to prevent further physical contact for the remainder of a 30-min session. Matched control rats were placed into a novel cage for the same duration (Chaijale et al, 2013; Wood et al, 2010).

\section{Attentional Set-Shifting Task}

Three days after the start of the experimental manipulation, rats were food restricted to $85 \%$ of their free-feeding weight. After the fifth day, rats were trained to perform an AST task (Figure 1a2; AST), which consisted of 1 day of habituation and 1 day of training as previously described (Birrell and Brown, 2000; Snyder, et al, 2012). Testing occurred 3 days after the last experimental manipulation and consisted of five discrimination stages: (1) SD, in which the rat chose to dig in one of two pots containing different digging materials to find food reward; (2) $\mathrm{CD}$, which required the same discrimination as before, however, the pots were scented with different odors, thereby providing irrelevant stimuli from a new dimension; (3) IDS, which changed exemplars from each dimension but maintained the same rule as SD so that the digging material was the relevant dimension; (4) REV, in which the reinforcement was associated with the alternate stimulus from the same dimension as IDS; (5) extradimensional shift (EDS), in which exemplars were changed and the relevant cue was changed to the other sensory dimension (odor). After the completion of each trial, rats were placed back into the start box. Six consecutive correct trials were required to proceed to the next stage. Behavior was videotaped and synchronized with the electrophysiological recording. Performance was quantified as the mean number of trials to reach criterion. In addition, analysis of error type during REV was quantified and broken down into perseverative and regressive errors as previously described (Cao et al, 2012).

\section{Electrophysiology}

The implanted multiwire array was connected by cables to the data acquisition system (Alpha Omega, Alpharetta, GA) and LC discharge rate was recorded before the start of the test and throughout testing as previously described (Curtis et al, 2012). LC discharge was recorded in the start box of the arena before starting each trial for at least $30 \mathrm{~s}$ (Figure 1a2; Baseline) and during task performance. The cable was connected at all times and rats were able to freely move during AST performance. Multiunit activity on all eight wires (channels) was monitored in real time and spike sorting of multiunit activity was done offline (Curtis et al, 2012).

\section{Analysis}

The WaveMark template-matching algorithm in the Spike2 software (Cambridge Electronic Design, CED, v7.09) was used to discriminate putative LC single-unit waveforms. A set of waveforms identified by the WaveMark template is verified as events from a single unit by the principal component analysis and associated autocorrelograms (Figure 1b1 and b2). Of 12 control and 10 stressed rats that were implanted with multichannel electrodes in the LC, welldiscriminated single units were obtained from 3 rats of each group by the time the rats were tested in the AST. For each channel with LC activity, two to five single units were usually discriminated. LC activity was typically isolated on two to four channels in an individual rat. LC discharge rates from all cells from each treatment group were averaged and 
a1

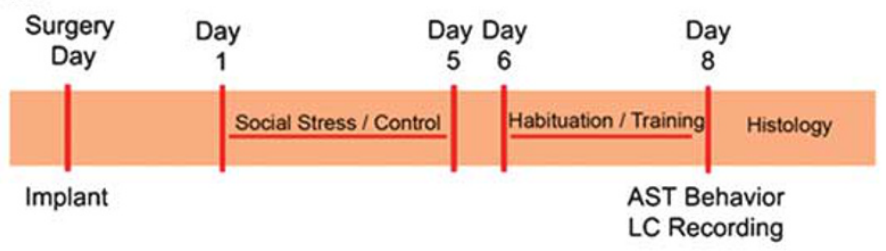

a2

AST Behavior

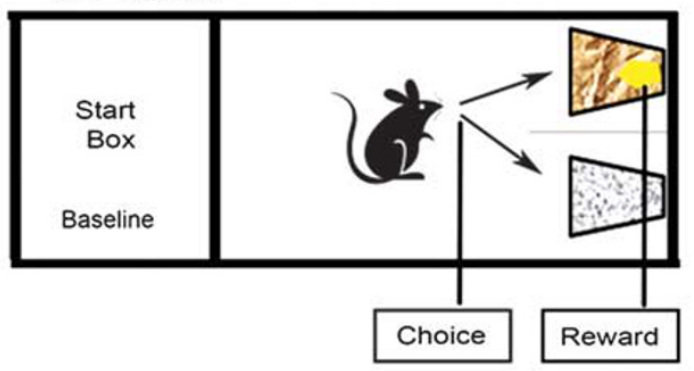

C

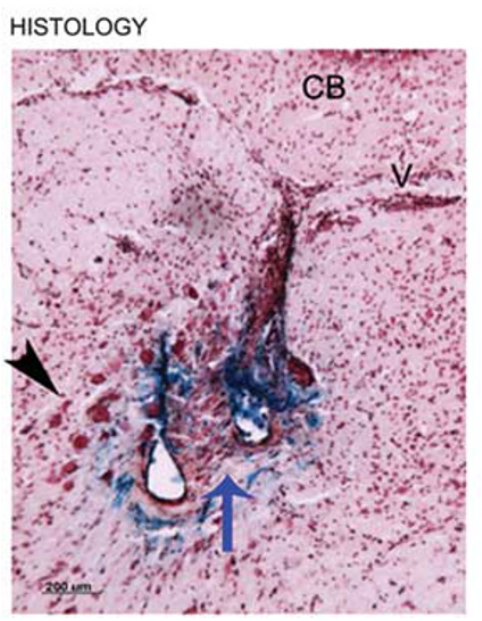

b1
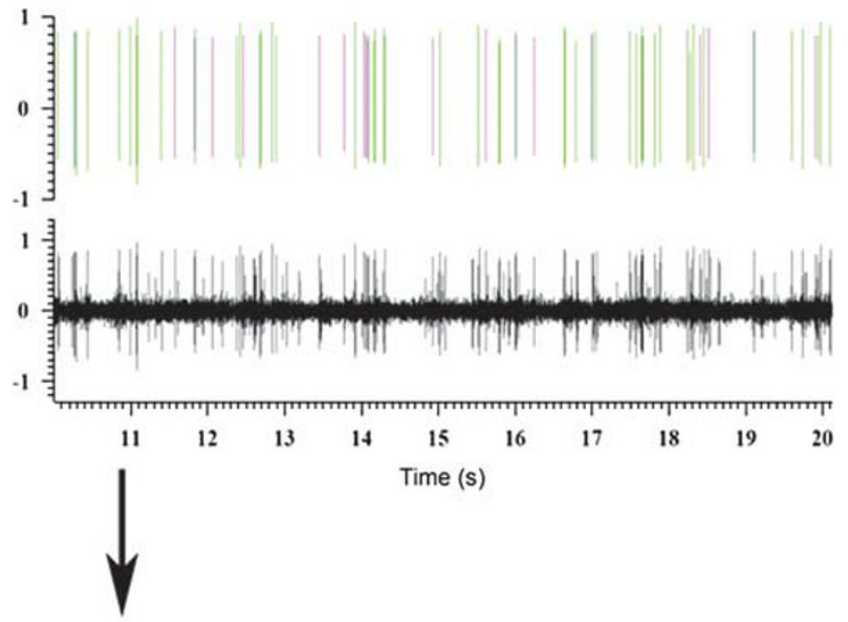

b2

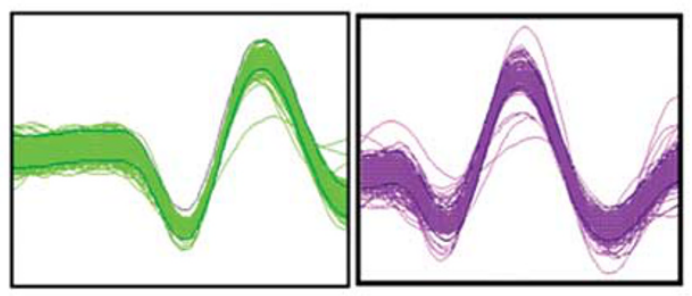

Figure I Schematic of experimental protocol. (a I) Timeline of surgery, stress, behavioral training, testing, and recording: Post surgery, rats were exposed to 5 days of social stress or control manipulation followed by habituation (Day 6) and training (Day 7) in the attentional set-shifting task (AST). Testing and locus coeruleus (LC) recording occurred on Day 8. (a2) Rats were placed in the start box and LC discharge was recorded before each trial of the task (baseline). The partition was removed for the animal to start the trial. The rat would travel to the other end of the arena where it chose to dig in one of two pots (separated by a partition) for food reward. LC activity was recorded before rats commit to a particular pot (choice), during the time between choice and reward, or reward omission (behavioral outcome), and after the behavioral outcome. (bl) The bottom trace shows raw waveforms from a single microwire located in the LC. The top trace shows sorted units from discriminated LC waveforms shown in b2. (b2) Waveforms are discriminated and analyzed off-line (depicted by the arrow). Waveforms from these units exhibited separable clusters when plotted in principal component analysis. (c) Photomicrograph illustrating the final placement of two of eight microwires in the LC as depicted by the blue arrow (black arrowhead, mesencephalic nerve 5; $\vee$, ventricle; $C B$, cerebellum).

plotted in 1-s. bins. The electrophysiological analysis focused on LC activity during IDS, REV, and EDS only, because behavior was identical between treatment groups for $S D$ and $C D$, and there were few incorrect trials in these stages. As cognitive processes and the neural circuits involved in IDS, REV, and EDS are different, LC activity during the individual stages was analyzed independently (Robbins and Roberts, 2007). Mean LC activity for all cells recorded in a treatment group (control $v s$ stress) during IDS, REV, or EDS was analyzed over the last six correct and the preceding six incorrect trials to correspond to the time that the rule was learned. In addition, for REV, activity was analyzed for the initial trials when the contingency was first reversed. For each trial, LC activity was analyzed at baseline (in the start box). Throughout the trial, LC activity was synchronized to the behavioral outcome (reward or reward omission). The average time between the choice (when animals committed to dig in one specific pot) and behavioral outcome was also determined. A one-way repeatedmeasures ANOVA was used to determine whether LC activity changed across time during the performance of a particular task stage over a period from $5 \mathrm{~s}$ before the choice to $10 \mathrm{~s}$ after behavioral outcome. Post-hoc comparisons (Student-Neuman-Keuls test) were done to determine at what time points during this period LC activity differed from the 5-s pre-choice baseline (SigmaStat, Systat 
software). In addition, to analyze whether stress induced phasic changes in reward-related neuronal activity, spike counts were reanalyzed in $50 \mathrm{~ms}$ bins for $2 \mathrm{~s}$ before and $2 \mathrm{~s}$ after the outcome. For each cell, the $z$-score was calculated by normalizing the mean spike count from each bin to the average activity of that unit during baseline. Heatmaps were generated to represent the mean $z$-score activity from each treatment group for the last six correct and preceding six incorrect trials. Values were smoothed with a 5-point averaging filter. In addition, histograms were generated to compare the activity of all cells from each treatment group across trials. Specifically, $z$-score values for the last six correct and preceding six incorrect trials were collapsed and plotted between a period of $2 \mathrm{~s}$ before and $2 \mathrm{~s}$ after reward or reward omission, and a paired Student's $t$-test was used for statistical comparison. Finally, to analyze LC phasic activation in response to the behavioral outcome during REV, the percentage of units firing simultaneously were calculated for every $50 \mathrm{~ms}$ bin. Results were plotted as heatmaps and time locked as $2 \mathrm{~s}$ before and $2 \mathrm{~s}$ after reward or discovery of reward omission (for incorrect trials). Heatmaps representing LC neurons from one control and one stressed rat were plotted with a set threshold such that bins would represent firing from at least $50 \%$ of the units. When at least $50 \%$ of the total number of cells from each rat fired within the same $50 \mathrm{~ms}$ bin, it was considered that LC activity was phasically activated. The non-parametric Wilcoxon signed-rank test was used to compare phasic activation before and after behavioral outcome.

\section{Histology}

Recording sites were localized by passing current $(10 \mu \mathrm{A}, 15 \mathrm{~s})$ through the wires followed by transcardial perfusion with $6 \%$ potassium ferrocyanide in $0.1 \mathrm{M}$ phosphate buffer to form a Prussian blue reaction (Curtis et al, 2012). After perfusion, brains were removed and stored in $-80^{\circ} \mathrm{C}$. Frozen coronal sections $(30 \mu \mathrm{m})$ were cut through the LC and thaw-mounted onto slides, stained with Neutral red, and the recording site was identified by microscopic visualization of the Prussian blue reaction product (Figure 1c).

\section{RESULTS}

Social Stress History Improves Performance in Certain Stages of the AST

Performance during most AST stages was comparable between control $(n=12)$ and stressed $(n=10)$ rats based on the mean number of trials to reach criterion (Figure 2a). However, stressed rats performed better during the IDS stage of the task (Figure 2a). A repeated-measures ANOVA indicated an interaction that was driven by IDS performance $(F(4,79)=7.0, p<0.01)$. Based on trials to criterion, REV performance was comparable between stressed and control groups. However, analysis of error type revealed that stressed rats made fewer perseverative errors $(p<0.05$, Student's $t$-test; Figure $2 \mathrm{~b}$ ). Although this appeared to be accompanied by an increase in regressive errors, this was not statistically significant $(p=0.3)$.

The duration between the time at which rats chose a pot and the time at which the outcome (reward or reward omission) was detected was calculated for the last six correct and incorrect trials of IDS, REV, and EDS. Rats with a history of social stress showed a greater delay between choice and outcome (11.0 $\pm 1.1 \mathrm{~s})$ compared with control rats $(7.1 \pm .5 \mathrm{~s}, p=0.02$, Student's $t$-test). Supplementary Figure S1 shows these data for individual stages and further divided into incorrect and correct trials. This effect is apparent in the graphs in Figure 3 (Task) that are synchronized to the outcome and also show the average time at which the choice occurred before the outcome for each group in each task.

\section{Social Stress History Renders LC Neurons Less Task Responsive and More Reward Responsive}

All control $(n=12)$ and stressed $(n=10)$ rats tested in the behavioral task described above were implanted with
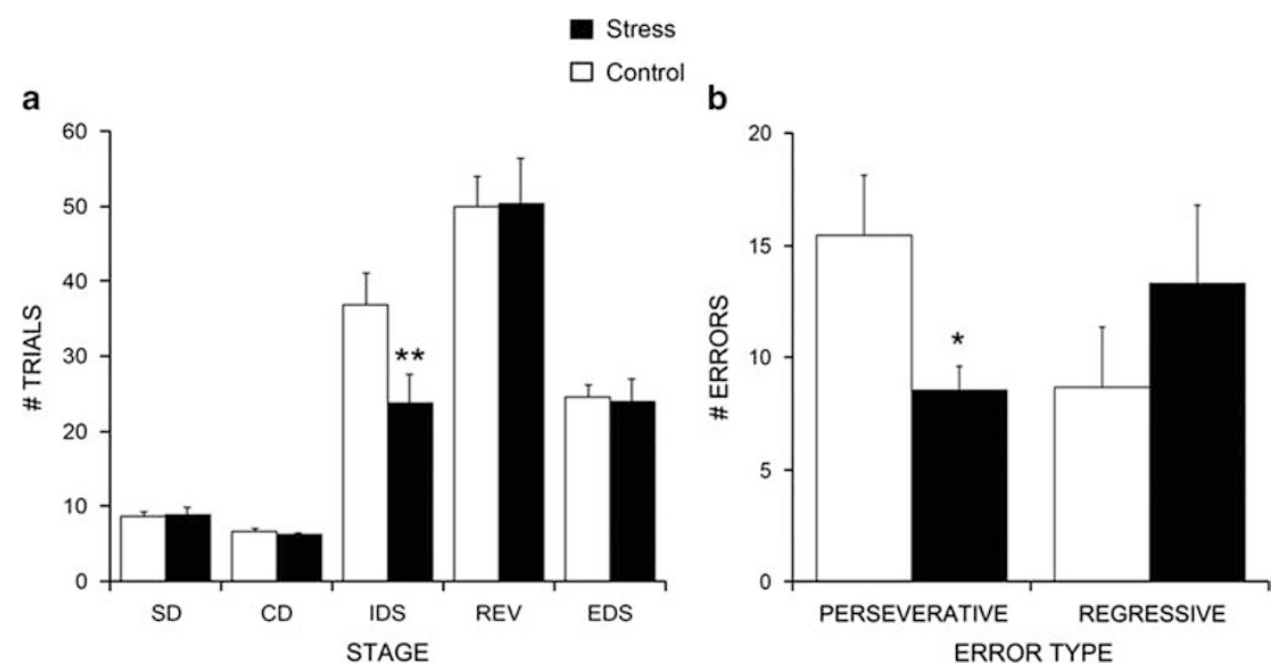

Figure 2 Attentional set-shifting performance of control and stressed rats. (a) The abscissa indicates the task stage: simple discrimination (SD), compound discrimination (CD), intradimensional shift (IDS), reversal learning (REV), and extradimensional shift (EDS). The ordinate indicates the number of trials to reach criterion. Bars represent the mean performance of 12 control (open bars) and 10 stressed (solid bars) rats \pm SEM. (b) The abscissa indicates the error type during reversal learning (REV) and the ordinate indicates the number of errors. Bars are mean $\pm \mathrm{SEM} * p<0.05$ and $* * *<0.0$ l. 

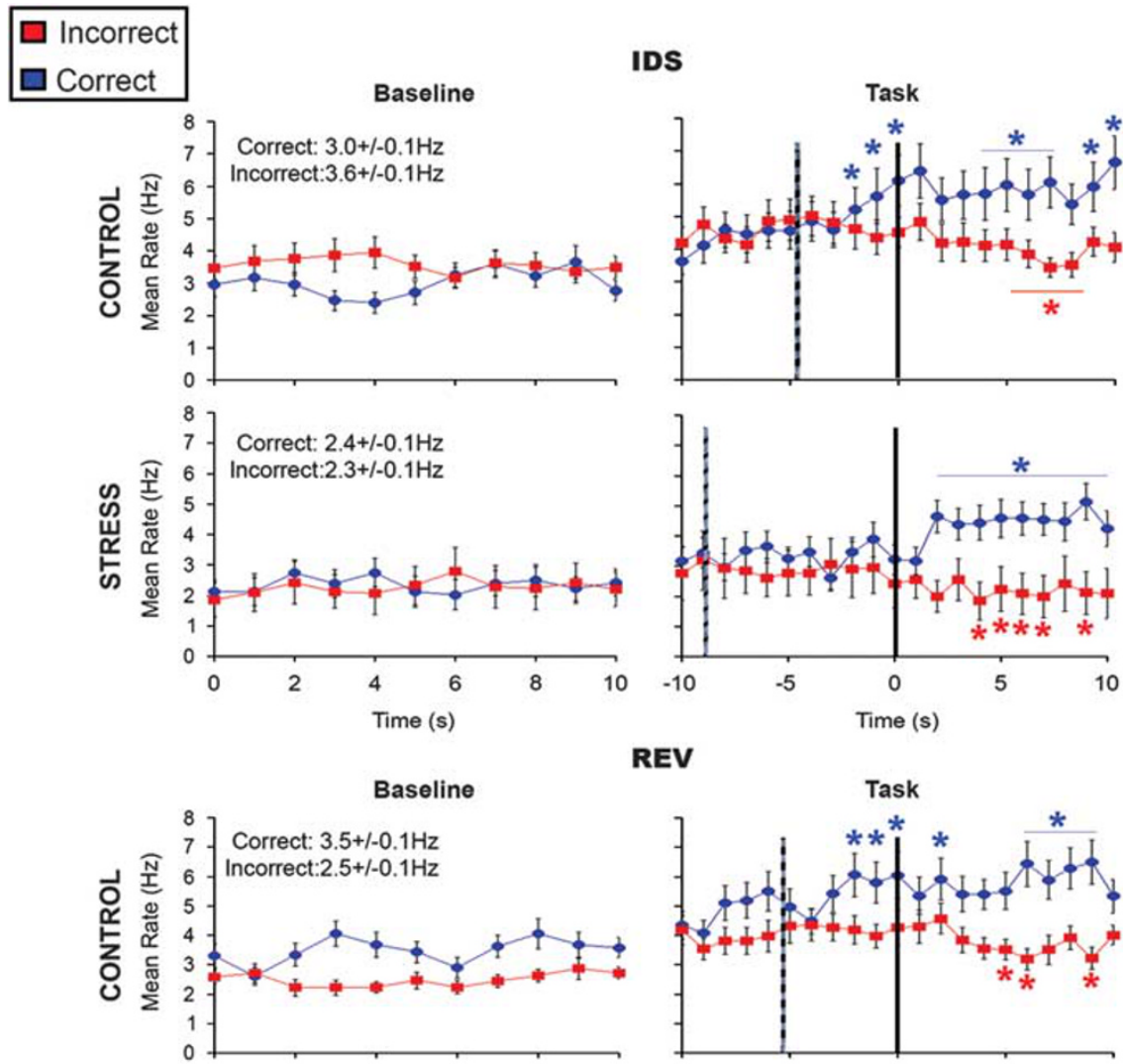

REV
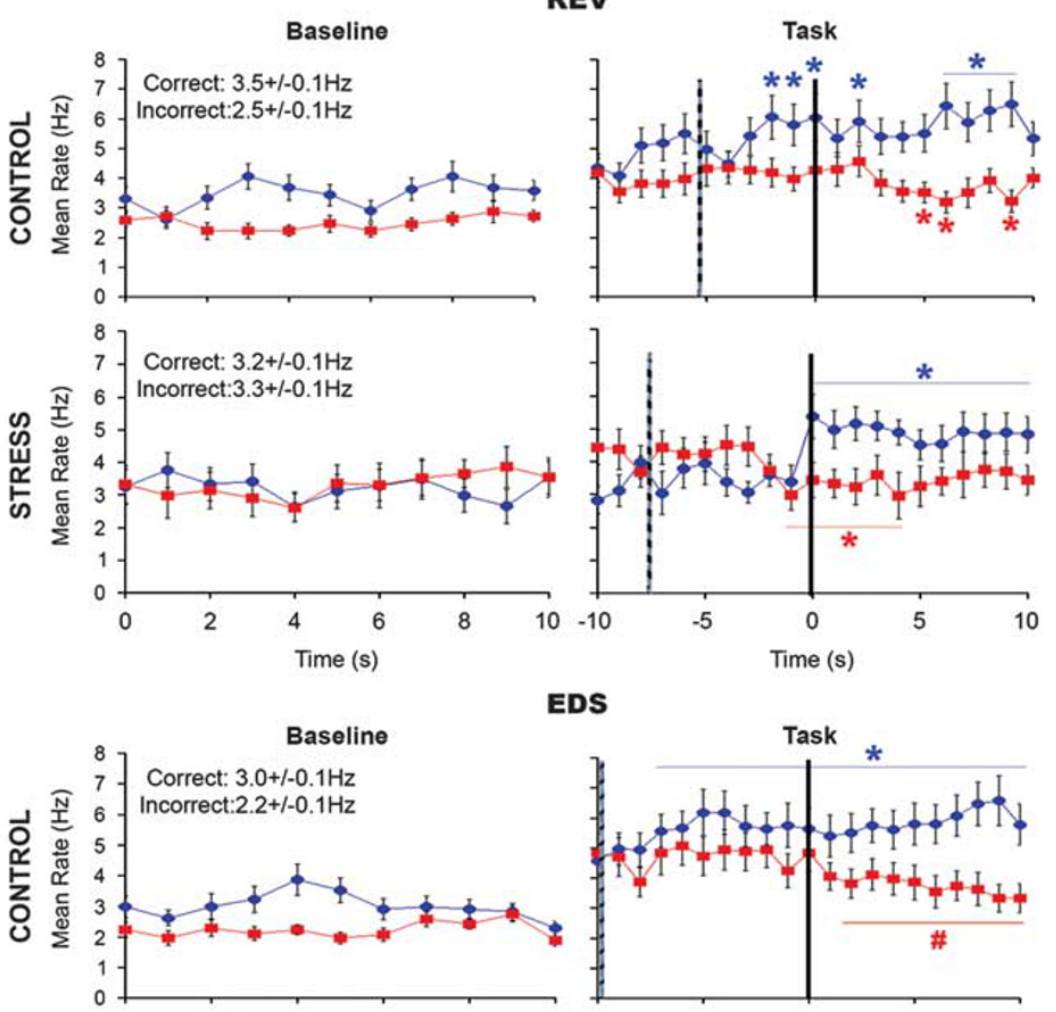

EDS
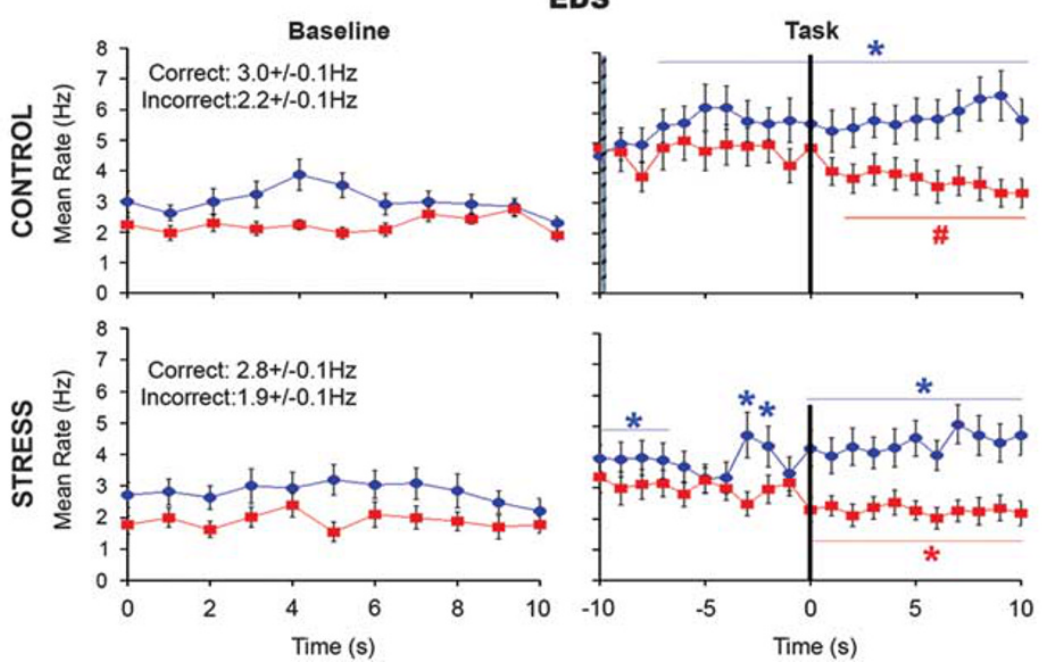

Figure 3 Locus coeruleus (LC) neuronal activity varies with behavior during intradimensional set shifting (IDS), reversal learning (REV), and extradimensional set-shifting (EDS) performance. Graphs on the left show IO s of LC spontaneous activity (Baseline), for control and stressed rats, before starting each correct (blue) or incorrect (red) trial. Mean LC discharge rates are provided in the insets. Graphs on the right represent mean LC activity during the last six correct (blue) and last six incorrect (red) trials, during IDS, REV, and EDS task performance (Task). LC activity is time synchronized with reward or reward omission (solid line at $t=0 \mathrm{~s}$ ). Dashed lines represent the average time at which the choice was made based on the mean duration between choice and reward. LC discharge is expressed as mean discharge rate across all units (mean \pm SEM). *p $<0.05$, Student-Neuman-Keuls. 
multichannel electrodes in the LC. Of these, 3 control (20 units) and 3 stressed (19 units) rats yielded well-discriminated single unit LC recordings after 5 days of social stress and 3 days of training and testing. Before testing, mean LC discharge rates were $3.9 \pm 0.4 \mathrm{~Hz}$ and $2.4 \pm 0.3 \mathrm{~Hz}$ for control and stressed rats, respectively ( $p<0.01$, Student's $t$-test). This was consistent with previous reports that baseline LC discharge rate is lower in rats with a history of social stress (Chaijale et al, 2013). During the last six correct and previous six incorrect trials baseline LC discharge recorded in the start box was relatively stable for both treatment groups and in all task stages (Figure 3, left-hand panels). A comparison of the baselines before starting the IDS stage showed no significant differences between correct and incorrect trials for either controls or stressed $(p>0.05$, Student's $t$-test) groups. In contrast, analysis of REV and EDS baselines demonstrated that LC neuronal activity was lower when control rats were about to start incorrect trials as compared with correct trials $(p<0.05$ for both REV and EDS). This effect was not observed in comparing LC baseline activity from stressed rats during any task stage (Figure 3 'Baseline').

For each task stage, LC neuronal activity varied with specific trial components in a way that differentiated treatment groups (Figure 3, Task). For control rats during correct IDS trials, LC activity increased $(\mathrm{F}(20,419)=6.9$, $p<0.001$, one-way repeated-measures ANOVA) and posthoc analysis indicated that this increase occurred during the period between choice and reward, compared with a 5-s pre-choice baseline, and did not increase further after the reward outcome (Figure 3, IDS, Task, Control). LC activity also varied during incorrect IDS trials $(\mathrm{F}(20,419)=3.5$, $p<0.001$, one-way repeated-measures ANOVA). However, during incorrect trials, LC discharge rate significantly decreased after discovery of reward omission (Figure 3, IDS, Task, Control). This decrease was only apparent at a few time points (6-8s after outcome).

Similar to that of control rats, LC activity of stressed rats changed during correct $(\mathrm{F}(23,455)=7.5, p<0.001$, one-way repeated-measures ANOVA) and incorrect $(\mathrm{F}(23,455)=2.4$, $p<0.001$, one-way repeated-measures ANOVA) trials of IDS performance (Figure 3 IDS, Task, Stress). However, in contrast to control rats, LC activity was not altered from the pre-choice baseline during the period between choice and reward for stressed rats during either correct or incorrect trials. Rather, LC activity of stressed rats were uniquely responsive to the behavioral outcome, being increased after reward for correct trials and inhibited after the discovery of reward omission for incorrect trials.

During REV, a similar distinction was apparent between control and stressed rats in the point at which LC activity was altered during performance. For control rats, LC discharge rates increased during correct REV trials $(\mathrm{F}(20,419)=4.9, p<0.001)$ and this occurred during the period between choice and reward as compared with a 5-s pre-choice baseline, and was not increased further in response to reward (Figure $3 \mathrm{REV}$, Task, Control). LC discharge rate decreased with the reward omission during incorrect trials $(\mathrm{F}(20,419)=2.9, p<0.001)$ but this was only statistically significant for a few time points between 5 and $9 \mathrm{~s}$ after the outcome. For stressed rats, LC neurons were clearly activated in response to reward $(F(22,398)=8.1$, $p<0.001)$ but not during the period between choice and reward (Figure 3, REV, Task, Stress). As for control rats, LC activity of stressed rats was decreased by reward omission during incorrect trials $(\mathrm{F}(20,398)=3.6, p<0.001)$ (Figure 3, Task, REV).

As LC responses to reward are related to predictability (Bouret and Sara, 2004), LC activity during the initial trials of REV, when contingencies were first reversed, was also examined. The relationship between LC activity and behavior during these early trials was similar to that seen in later trials (Figure 4). LC discharge rates of control rats increased during performance $(\mathrm{F}(20,419)=6.4, p<0.001)$, and this occurred specifically during the choice-to-reward period and remained increased compared with the 5-s prechoice baseline. LC discharge rates of stressed rats were also increased during task performance but only after reward $(\mathrm{F}(23,455)=6.4, p<0.001)$. During the incorrect trials, LC activity of control rats increased during choice-to-reward period $(\mathrm{F}(20,419)=2.5, p<0.001)$ but did not change after reward omission. For stressed rats during incorrect early REV trials, LC activity decreased just before the discovery of reward omission and this was maintained for a few additional seconds $(\mathrm{F}(23,455)=2.6, p<0.001)$.

Similar to the previous stages, during EDS LC neuronal activity of control rats changed during task performance $(\mathrm{F}(24,499)=6.7, p<0.001)$ and was specifically increased within the period between choice and reward compared with a 5-s pre-choice baseline and did not increase further with reward (Figure 3 EDS, Task, Control). There was only a trend for LC activity to be inhibited by reward omission during incorrect trials $(\mathrm{F}(24,499)=1.5, p=0.07)$. Notably, LC neurons of stressed rats responded in a similar manner as control rats during EDS in that they were activated during the choice-to-reward component compared with the 5 -s pre-choice baseline and were not further responsive to reward during $\operatorname{EDS}(\mathrm{F}(29,569)=6.3, p<0.001)$. In addition, the neurons were inhibited by reward omission during incorrect trials $(\mathrm{F}(29,590)=4.6, p<0.001)$ (Figure 3 EDS, Task, Stress).

\section{LC Neurons of Stressed Rats are Phasically Activated by Reward During REV}

LC discharge was further examined across a more discrete time frame $( \pm 2 s)$ around the behavioral outcome to determine whether LC neurons were phasically activated by reward. Figure 5a shows LC activity for controls and stressed rats during correct and incorrect REV trials with respect to behavioral outcome expressed as $z$-score transformations normalized to baseline rates (see Figure 3 ). For REV, the results seen within this discrete time frame reflect those seen in the longer time frame in Figure 3. LC activity of control rats is activated above baseline and remains constant even after reward. For stressed rats, LC activity is not increased before reward but is abruptly increased after reward for about $800 \mathrm{~ms} \quad(p<0.05$, Student's paired $t$-test). The absence of reward had no immediate consequence on LC discharge of either stressed or control rats (Figure 5a). Phasic LC activation elicited by reward did not occur during IDS or EDS components of the task. 

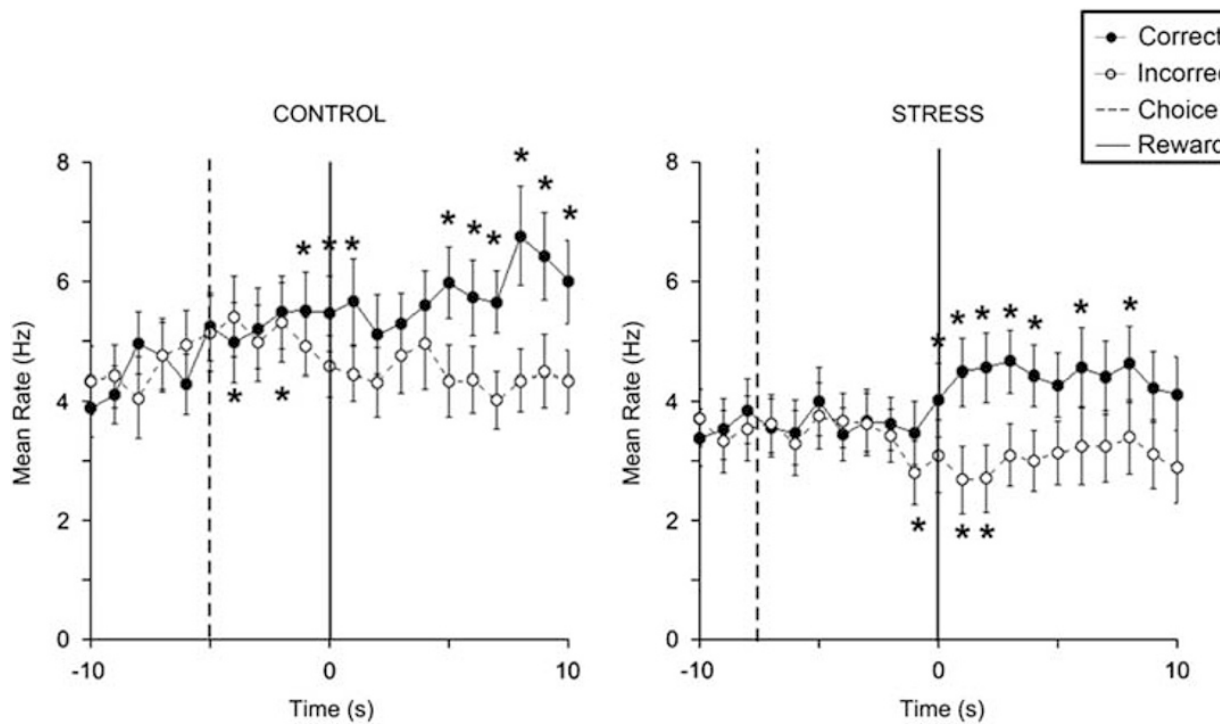

Figure 4 Mean firing rate of locus coeruleus (LC) neurons from control and stress rats during early trials of reversal learning (REV). Graphs represent mean $\mathrm{LC}$ mean firing rate $(\mathrm{Hz})$ during the first six correct (solid circles) and first six incorrect (open circles) trials of REV. Discharge rate is time-synchronized to the reward point (solid line, $t=0 \mathrm{~s}$ ). Dashed lines indicate the average time before outcome that the choice occurred. LC discharge is expressed as mean discharge rate across all units (mean \pm SEM). ${ }^{*} p<0.05$, Student-Neuman-Keuls.

To visualize phasic activation on a trial-by-trial basis, heatmaps of the normalized ( $z$-score) LC activity recorded during the last six correct trials and previous six incorrect trials during REV performance were generated (Figure 5a and $b$, respectively). The heatmaps reflect the results depicted in the histograms and there was no apparent effect of trial order.

Differences in reward-related phasic LC activity during REV were also apparent in heat maps illustrating the percentage of the total number of cells that fired within the same $50 \mathrm{~ms}$ bin, (Figure $5 \mathrm{~b}$ ). LC phasic activation associated with reward is apparent in the stressed rat $(p<0.05$, paired Student's $t$-test; Figure 5, Stress, Correct) but not the control rat (Figure 5, Control, Correct). There are no apparent changes in LC activity by reward omission.

\section{DISCUSSION}

Although stress has generally been associated with impaired cognitive function, here a history of repeated social stress was associated with better IDS performance and decreased perseverative responding during REV. Importantly, social stress changed the relationship between LC discharge rate and behavior. In agreement with previous studies (Bouret and Sara, 2004; Clayton et al, 2004), LC neurons of control but not stressed rats were task-responsive for correct trials, exhibiting greater discharge rates between choice and the behavioral outcome. This was associated with a shorter time to obtain reward after making the decision for control compared with stressed rats. Social stress history shifted LC neurons from being task responsive to being reward responsive during correct trials. LC responses to reward were unrelated to prediction error, because they did not habituate during later trials in which reward would be expected. LC neurons of both experimental groups were inhibited by reward omission in incorrect trials. These results suggest that social stress increases reward salience and impairs encoding of predictability by LC neurons. Stress-induced changes in the relationship between LC neuronal activity and aspects of goal-directed behavior could facilitate certain types of learning and protect against perseverative behavior. However, this stress-induced plasticity may also be expressed as exaggerated arousal responses to behavioral consequences that are characteristic of psychiatric disorders, such as PTSD. The increased reward salience is also seen in humans exposed to stress and this may have a role in the link between stress and addictive behaviors (Mather and Lighthall, 2012).

\section{Repeated Social Stress Enhances Some Aspects of Cognitive Performance}

Both acute and chronic stressors have been demonstrated to produce impairments of executive function and this has been linked to effects on the prefrontal cortex (PFC) (Arnsten, 2009; Holmes and Wellman, 2009). Thus, acute tail pinch, chronic restraint, and chronic unpredictable stress impair attention set-shifting or strategy-shifting performance (Bondi et al, 2008; Butts et al, 2013; Jett and Morilak, 2013; Liston et al, 2006). However, this effect does not generalize across all stress conditions. For example, chronic cold stress selectively impaired REV (Lapiz-Bluhm et al, 2009). Moreover, acute restraint and repeated (but not a single) swim stress enhanced REV (Graybeal et al, 2011; Thai et al, 2013). It has been suggested that by decreasing the influence of the ventromedial PFC, stressors bias the regulation of cognitive processes toward systems that favor alternate strategies, such as the orbitofrontal cortex and dorsolateral striatum, and this may explain the enhancement of REV by some stress conditions (Graybeal et al, 2011). In this light, in the present study a history of repeated 
a
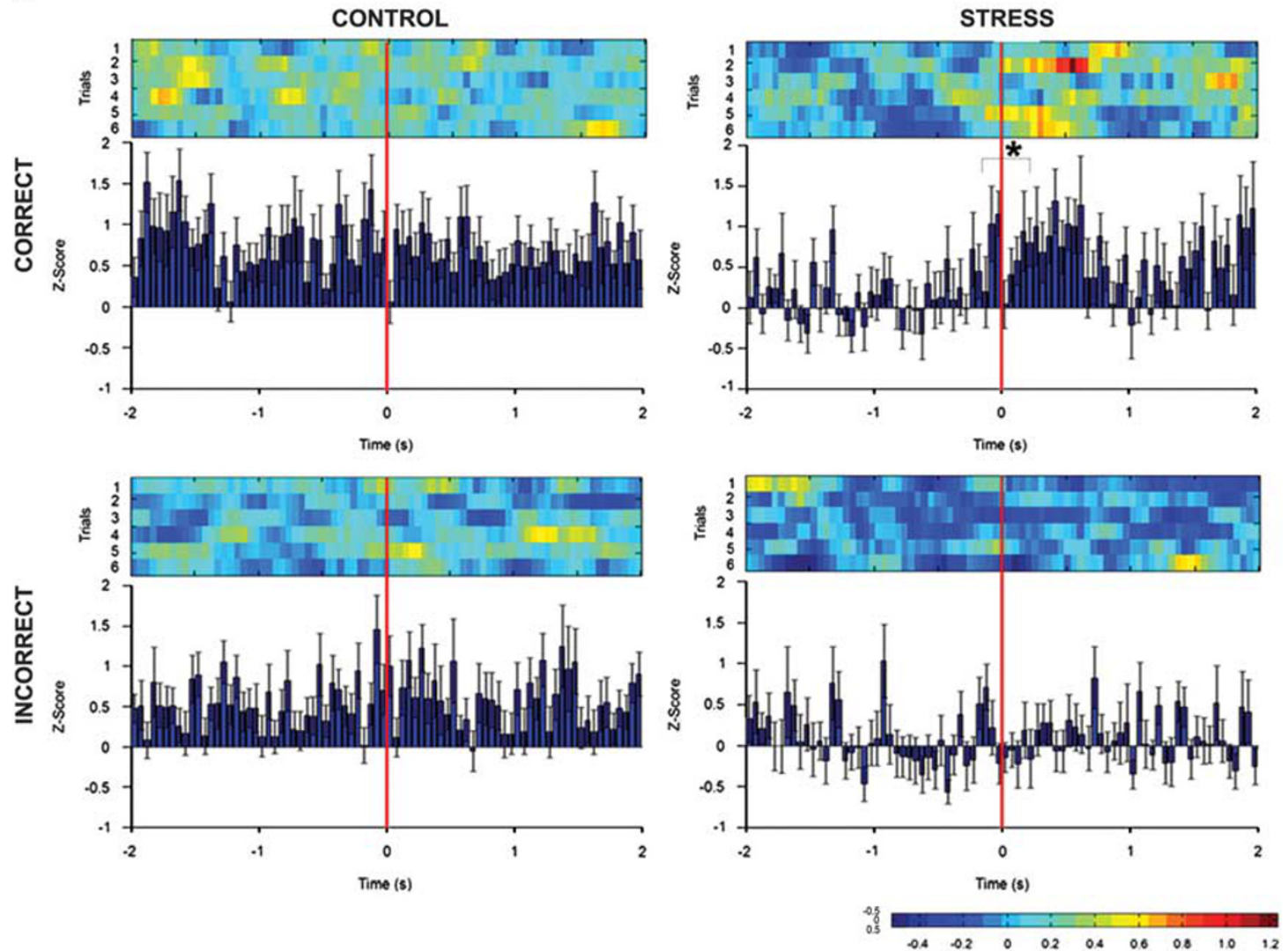

b
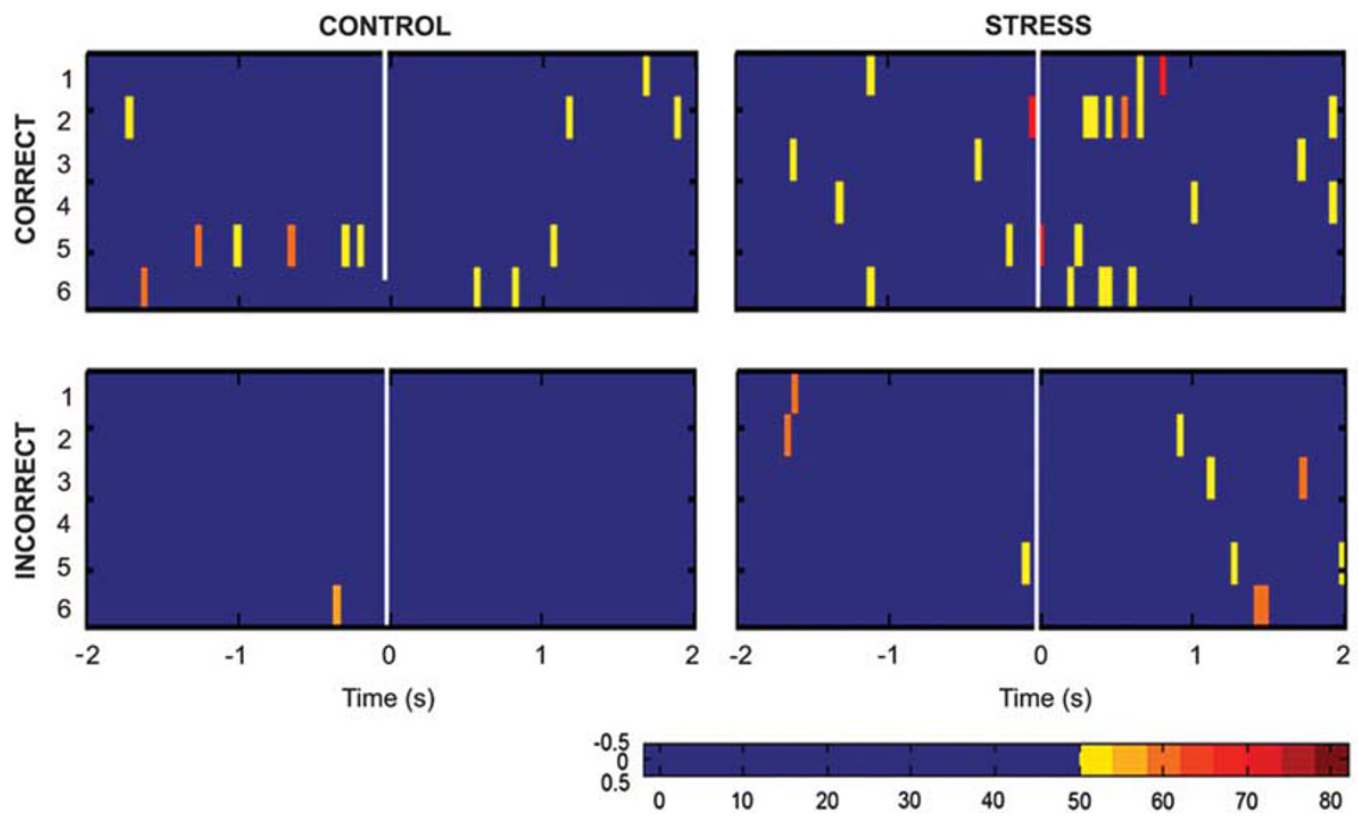

Figure 5 Stress increased phasic locus coeruleus (LC) responses to reward during reversal learning (REV) performance. (a) Heatmaps represent LC activity from control and stressed rats during the last six correct or incorrect trials. Histograms represent LC neuronal activity expressed as z-scores normalized to the baseline for REV during the same trials collapsed. Histograms are time synchronized to reward or reward omission (red line at $t=0 \mathrm{~s}$ ). In stressed rats, LC neuronal activity was phasically increased following reward (* $p<0.05$, Student's paired $t$-test). Phasic LC activation was not elicited by reward omission in stressed rats. It also did not occur in control rats in response to reward or reward omission. (b) Heatmaps represent the percentage of phasically activated LC neurons in response to reward (correct) or reward omission (incorrect) during REV. Heatmaps show neuronal activity from a representative control and stressed rat ( $n=9$ cells, each rat), during REV trials (TI-T6, last six correct or the preceding six incorrect trials). Plots are time locked at reward or reward omission (white line at $0 \mathrm{~s}$ ). For each correct and incorrect trial, burst activity was measured every 50 ms bin and expressed as the percentage of neurons that fired in each bin. Neurons from stressed rats exhibit more phasic discharge after reward ( $p<0.05$, Student's paired $t$-test), whereas controls do not $(p=0.7)$. 
social stress did not impair performance in any component of the task and, unexpectedly, enhanced IDS and REV performance. Notably, excessive activation of the LC-NE system has been implicated in stress-induced cognitive impairments (Birnbaum et al, 1999; Jett and Morilak, 2013). However, this does not occur in rats with repeated social stress history (Chaijale et al, 2013 and the present study). Rather, LC discharge rates are inhibited compared with controls. This effect is evident for several days after the final stress exposure and it is due to an engagement of endogenous opioid afferents to the LC and downregulation of CRF receptors (Chaijale et al, 2013). This relative inhibition of LC discharge was reproduced in the current study as indicated by the mean LC discharge rates recorded in home cages before testing in the AST. Stress-induced regulation of the LC system by opioids can account for the enhancement of IDS performance and the decreased number of perseverative errors during REV, because in humans acute morphine administration had similar positive effects on IDS and REV (Quednow et al, 2008). In line with the model of competing systems (Graybeal et al, 2011) the lower LC activity in stressed rats may facilitate a shift toward systems that favor IDS and REV. Importantly, other studies demonstrated that chronic resident-intruder stress does not impair and, in some cases, improves performance in memory tasks (Buwalda et al, 2005). Together, the results underscore the likelihood that different stressors will have unique cognitive consequences because they differentially affect the underlying circuits.

\section{Repeated Social Stress Changes the Relationship between LC Activity and Behavior}

Previous studies of LC neuronal activity in monkeys and rats during behavior directed by discrete cues in operant procedures indicated that LC neurons are task responsive and not reward responsive, being phasically activated following conditioned stimuli predicting reward and preceding reward (Bouret and Sara, 2004; Clayton et al, 2004). Although initially LC neurons are activated by unpredicted reward, this response habituates and shifts toward taskrelated responses when the outcome becomes predictable. The shift from reward- to task-related activation may facilitate the development of conditioned reinforcement (Schultz and Dickinson, 2000). The task used in the present study differs from the operant procedures in requiring discrimination between cues that are simultaneously and continuously present, rather than being discretely presented so that they would not be expected to elicit a phasic response. These settings may better represent conditions under which human decisions are made because stimuli that provide information about behavioral response outcomes are unlikely to be briefly presented stimuli. Regardless of the procedural differences, the current findings in control rats are consistent with previous studies in suggesting that LC neurons are task responsive at least for correct trials, because for this group LC activity reliably increased after the decision and before the reward period. Previous studies suggested that task-related LC activity facilitates the behavior (Clayton et al, 2004). Consistent with this, LC neurons of stressed rats were not task responsive and these rats took longer to dig and obtain reward.

Social stress history rendered LC neurons reward responsive during IDS and REV, and impaired habituation of LC activation by expected reward and a shift to taskrelated LC activation. The ability of stress to promote reward-related LC activation is consistent with an increase in reward salience and in agreement with human studies showing that stress (including psychosocial stress) increases reward salience (Lighthall et al, 2013; Mather and Lighthall, 2012; Petzold et al, 2010). Interestingly, evidence from our laboratory and others suggests that a history of stress also increases the salience of noxious stimuli based on LC responses (see below). The finding that reward-related LC activation does not habituate as reward becomes more predictable suggests that neural encoding of prediction is impaired. Neuronal encoding of prediction error, the difference between the predicted and actual occurrence of an outcome, is a basis for learning (Schultz and Dickinson, 2000). LC neurons adapt to predicted reward by shifting responses from the reward to the task (Bouret and Sara, 2004; Clayton et al, 2004). The findings that reward-related LC activation did not habituate or transfer to task-related activation suggests that learning at the level of these neurons was maintained in premature state in which salience of the outcome, but not the task, is high. This could explain the decreased perseveration during REV in stressed rats. LC neurons were tonically inhibited by reward omission for both groups in all task phases. This inhibition, in concert with reward-related LC activation in stressed rats, would be expected to augment learning. Notably, the effects of stress were apparent during IDS and REV but not EDS, suggesting that alternate circuits are engaged during EDS that regulate LC activity with different consequences, perhaps allowing for task-related LC activation to occur.

\section{Potential Cellular Mechanisms Underlying Social Stress-Induced LC Plasticity}

Increased reward-related LC activity seen in the present study may be mechanistically related to enhanced footshock-evoked LC activity that has been reported with social stress and chronic cold stress (Bingham et al, 2011; Finlay et al, 1997). Importantly, this is also seen with the single prolonged stress model of PTSD, underscoring the potential role of this effect in symptoms of PTSD (George et al, 2013). Enhanced responses of LC neurons to salient stimuli could result from increased glutamatergic function in the LC, because excitatory amino acid afferents are major mediators of LC activation by discrete stimuli (Ennis and Aston-Jones, 1988). Alternatively, changes in endogenous opioid regulation of the LC may underlie these effects. Although opiates generally inhibit spontaneous LC discharge, in moderate doses they increase the signal-to-noise ratio of LC sensory responses, an effect that would favor enhanced responses to salient stimuli (Valentino and Foote, 1988).

\section{Clinical Implications}

Social stress-induced enhancement of LC responses to reward may be advantageous to learning processes. However, these effects may also underlie some of the symptoms 
of stress-related psychiatric disorders. For example, accentuated activation of the LC-NE system by rewarding stimuli such as food or drugs of abuse would be predicted to increase the arousal response to those stimuli and could underlie the role of stress in obesity and/or substance abuse. The effect of social stress to increase LC responses to reward taken with its ability to induce a cellular state of opioid dependence (Chaijale et al, 2013) converge to make subjects with a history of social stress particularly vulnerable to opiate abuse. Finally, the LC is poised to mediate the arousal component of emotional responses through its connections with the central amygdalar nucleus. As exaggerated emotional arousal in response to behavioral outcomes is characteristic of PTSD, the present results provide a mechanism by which repeated social stress contributes to this disease.

\section{FUNDING AND DISCLOSURE}

The authors declare no conflict of interest.

\section{ACKNOWLEDGEMENTS}

We acknowledge Dr Seema Bhatnagar for helpful comments on the research and manuscript. This work was supported by grants from the Defense Advanced Research Projects Agency (DARPA 58077 LSDRP) and PHS grants DA09082, T32NS007413 and T32MH14654.

\section{REFERENCES}

Arnsten AF (2009). Stress signalling pathways that impair prefrontal cortex structure and function. Nat Rev Neurosci 10: $410-422$.

Aston-Jones G, Bloom FE (1981a). Norepinephrine-containing locus coeruleus neurons in behaving rats exhibit pronounced responses to non-noxious environmental stimuli. J Neurosci 1: 887-900.

Aston-Jones G, Bloom FE (1981b). Activity of norepinephrinecontaining locus coeruleus neurons in behaving rats anticipates fluctuations in the sleep-waking cycle. J Neurosci 1: 876-886.

Aston-Jones G, Cohen JD (2005). An integrative theory of locus coeruleus-norepinephrine function: adaptive gain and optimal performance. Annu Rev Neurosci 28: 403-450.

Berridge CW, Waterhouse BD (2003). The locus coeruleusnoradrenergic system: modulation of behavioral state and state-dependent cognitive processes. Brain Res Brain Res Rev 42: 33-84.

Bingham B, McFadden K, Zhang X, Bhatnagar S, Beck S, Valentino R (2011). Early adolescence as a critical window during which social stress distinctly alters behavior and brain norepinephrine activity. Neuropsychopharmacology 36: 896-909.

Birnbaum S, Gobeske KT, Auerbach J, Taylor JR, Arnsten AF (1999). A role for norepinephrine in stress-induced cognitive deficits: alpha-1-adrenoceptor mediation in the prefrontal cortex. Biol Psychiatry 46: 1266-1274.

Birrell JM, Brown VJ (2000). Medial frontal cortex mediates perceptual attentional set shifting in the rat. J Neurosci 20: 4320-4324.

Bondi CO, Rodriguez G, Gould GG, Frazer A, Morilak DA (2008). Chronic unpredictable stress induces a cognitive deficit and anxiety-like behavior in rats that is prevented by chronic antidepressant drug treatment. Neuropsychopharmacology 33: 320-331.
Bouret S, Sara SJ (2004). Reward expectation, orientation of attention and locus coeruleus-medial frontal cortex interplay during learning. Eur J Neurosci 20: 791-802.

Bouret S, Sara SJ (2005). Network reset: a simplified overarching theory of locus coeruleus noradrenaline function. Trends Neurosci 28: 574-582.

Butts KA, Floresco SB, Phillips AG (2013). Acute stress impairs set-shifting but not reversal learning. Behav Brain Res 252: 222-229.

Buwalda B, Kole MH, Veenema AH, Huininga M, de Boer SF, Korte SM et al (2005). Long-term effects of social stress on brain and behavior: a focus on hippocampal functioning. Neurosci Biobehav Rev 29: 83-97.

Cao AH, Yu L, Wang YW, Wang JM, Yang LJ, Lei GF (2012). Effects of methylphenidate on attentional set-shifting in a genetic model of attention-deficit/hyperactivity disorder. Behav Brain Funct 8: 10.

Chaijale NN, Curtis AL, Wood SK, Zhang XY, Bhatnagar S, Reyes BA et al (2013). Social stress engages opioid regulation of locus coeruleus norepinephrine neurons and induces a state of cellular and physical opiate dependence. Neuropsychopharmacology 38: 1833-1843.

Chrousos GP, Gold PW (1992). The concepts of stress and stress system disorders. Overview of physical and behavioral homeostasis. JAMA 267: 1244-1252.

Clayton EC, Rajkowski J, Cohen JD, Aston-Jones G (2004). Phasic activation of monkey locus ceruleus neurons by simple decisions in a forced-choice task. J Neurosci 24: 9914-9920.

Curtis AL, Leiser SC, Snyder K, Valentino RJ (2012). Predator stress engages corticotropin-releasing factor and opioid systems to alter the operating mode of locus coeruleus norepinephrine neurons. Neuropharmacology 62: 1737-1745.

de Kloet ER, Joels M, Holsboer F (2005). Stress and the brain: from adaptation to disease. Nat Rev Neurosci 6: 463-475.

Ennis M, Aston-Jones G (1988). Activation of locus coeruleus from nucleus paragigantocellularis: a new excitatory amino acid pathwway in brain. J Neurosci 8: 3644-3657.

Finlay JM, Jedema HP, Ravinovic AD, Mana MJ, Zigmond MJ, Sved AF (1997). Impact of corticotropin-releasing hormone on extracellular norepinephrine in prefrontal cortex after cold stress. J Neurochem 69: 144-150.

Foote SL, Aston-Jones G, Bloom FE (1980). Impulse activity of locus coeruleus neurons in awake rats and monkeys is a function of sensory stimulation and arousal. Proc Natl Acdad Sci USA 77: 3033-3037.

George SA, Knox D, Curtis AL, Aldridge JW, Valentino RJ, Liberzon I (2013). Altered locus coeruleus-norepinephrine function following single prolonged stress. Eur J Neurosci 37: 901-909.

Gold PW, Chrousos GP (2002). Organization of the stress system and its dysregulation in melancholic and atypical depression: high vs low CRH/NE states. Mol Psych 7: 254-275.

Graybeal C, Feyder M, Schulman E, Saksida LM, Bussey TJ, Brigman JL et al (2011). Paradoxical reversal learning enhancement by stress or prefrontal cortical damage: rescue with BDNF. Nat Neurosci 14: 1507-1509.

Holmes A, Wellman CL (2009). Stress-induced prefrontal reorganization and executive dysfunction in rodents. Neurosci Biobehav Rev 33: 773-783.

Jett JD, Morilak DA (2013). Too much of a good thing: blocking noradrenergic facilitation in medial prefrontal cortex prevents the detrimental effects of chronic stress on cognition. Neuropsychopharmacology 38: 585-595.

Lapiz-Bluhm MD, Soto-Pina AE, Hensler JG, Morilak DA (2009). Chronic intermittent cold stress and serotonin depletion induce deficits of reversal learning in an attentional set-shifting test in rats. Psychopharmacology (Berl) 202: 329-341. 
Lighthall NR, Gorlick MA, Schoeke A, Frank MJ, Mather M (2013). Stress modulates reinforcement learning in younger and older adults. Psychol Aging 28: 35-46.

Liston C, Miller MM, Goldwater DS, Radley JJ, Rocher AB, Hof PR et al (2006). Stress-induced alterations in prefrontal cortical dendritic morphology predict selective impairments in perceptual attentional set-shifting. J Neurosci 26: 7870-7874.

Mather M, Lighthall NR (2012). Both risk and reward are processed differently in decisions made under stress. Curr Dir Psychol Sci 21: 36-41.

McEwen BS (1998). Stress, adaptation, and disease. Allostasis and allostatic load. Ann N Y Acad Sci 840: 33-44.

Miczek KA (1979). A new test for aggression in rats without aversive stimulation: differential effects of d-amphetamine and cocaine. Psychopharmacology (Berl) 60: 253-259.

Petzold A, Plessow F, Goschke T, Kirschbaum C (2010). Stress reduces use of negative feedback in a feedback-based learning task. Behav Neurosci 124: 248-255.

Quednow BB, Csomor PA, Chmiel J, Beck T, Vollenweider FX (2008). Sensorimotor gating and attentional set-shifting are improved by the mu-opioid receptor agonist morphine in healthy human volunteers. Int J Neuropsychopharmacol 11: 655-669.

Robbins TW, Roberts AC (2007). Differential regulation of frontoexecutive function by the monoamines and acetylcholine. Cereb Cortex 17(Suppl 1): i151-i160.

Sara SJ (2009). The locus coeruleus and noradrenergic modulation of cognition. Nature Rev. Neuroscience 10: 211-223.

Schultz W, Dickinson A (2000). Neuronal coding of prediction errors. Ann Rev Neurosci 23: 473-500.

Snyder K, Wang WW, Han R, McFadden K, Valentino RJ (2012). Corticotropin-releasing factor in the norepinephrine nucleus, locus coeruleus, facilitates behavioral flexibility. Neuropsychopharmacology 37: 520-530.

Southwick SM, Bremner JD, Rasmusson A, Morgan CA 3rd, Arnsten A, Charney DS (1999). Role of norepinephrine in the pathophysiology and treatment of posttraumatic stress disorder. Biol Psychiatry 46: 1192-1204.

Thai CA, Zhang Y, Howland JG (2013). Effects of acute restraint stress on set-shifting and reversal learning in male rats. Cogn Affect Behav Neurosci 13: 164-173.

Valentino RJ, Foote SL (1987). Corticotropin-releasing factor disrupts sensory responses of brain noradrenergic neurons. Neuroendocrinology 45: 28-36.

Valentino RJ, Wehby RG (1988). Corticotropin-releasing factor: Evidence for a neurotransmitter role in the locus coeruleus during hemodynamic stress. Neuroendocrinology 48: 674-677.

Valentino RJ, Foote SL (1988). Corticotropin-releasing factor increases tonic but not sensory-evoked activity of noradrenergic locus coeruleus neurons in unanesthetized rats. J Neurosci 8: 1016-1025.

Valentino RJ, Van Bockstaele E (2008). Convergent regulation of locus coeruleus activity as an adaptive response to stress. Eur J Pharmacol 583: 194-203.

Wong ML, Kling MA, Munson PJ, Listwak S, Licinio J, Prolo P et al (2000). Pronounced and sustained central hypernoradrenergic function in major depression with melancholic features: relation to hypercortisolism and corticotropin-releasing hormone. Proc Natl Acad Sci USA 97: 325-330.

Wood SK, Walker HE, Valentino RJ, Bhatnagar S (2010). Individual differences in reactivity to social stress predict susceptibility and resilience to a depressive phenotype: role of corticotropin-releasing factor. Endocrinology 151: 1795-1805.

Supplementary Information accompanies the paper on the Neuropsychopharmacology website (http://www.nature.com/npp) 